\title{
Logique, mais depuis longtemps attendu: le gène impliqué dans le syndrome de Bloom a une activité ADN hélicase
}

Le syndrome de Bloom (BS) est une des réparatoses des plus spectaculaires puisqu'il est loisible d'observer dans les cellules somatiques des malades en culture in vitro une quantité extraordinaire d'échanges de chromatides sœurs (SCE), phénomène qui confirme biologiquement le diagnostic. Il s'agit d'une maladie redoutable car, chez ces sujets de petite taille avec immunodéficience et hypersensibilité au soleil, la prédisposition à tous les types de cancers est énorme.

La recherche du gène fut laborieuse, d'où l'extrême discrétion sur ce syndrome lorsque fut abordé le thème des réparatoses dans nos colonnes [1, 2]. Maladie récessive heureusement peu fréquente, il fallut les efforts conjugués de James German aux États-Unis d'Eberhard Passarge en Europe pour en établir un registre [3] et avoir les moyens d'entreprendre la recherche du gène $B L M$. Grâce au clonage positionnel et à l'étude de familles consanguines [4], celui-ci fut localisé en 15q26.1, dans une région comprenant aussi le proto-oncogène FES dont la responsabilité dans le BS fut rapidement exclue. Le gène, dont le rôle est assurément fondamental dans la transcription de l'ADN, vient enfin d'être isolé grâce à une stratégie originale et très efficace dans ce cas: la cartographie du point de recombinaison somatique [5]. La méthode est facile à comprendre. Dans les cultures de lymphoblastes des sujets BS, il n'est pas rare de constater une normalisation des SCE. L'hypothèse la plus vraisemblable pour expliquer ce phénomène était la suppression d'une des deux mutations par recombinaison somatique chez des malades hétérozygotes composites, n'ayant pas hérité de la même mutation de chacun de leurs parents. Cette hypothèse fut vérifiée à l'aide de marqueurs polymorphiques de la région critique. La comparaison entre la lignée initiale et la population cellulaire normalisée montra en effet toujours la même modification: les marqueurs distaux deviennent homozygotes alors que les marqueurs proximaux restent hétérozygotes (figure 1). Il ne restait plus alors qu'à explorer cette région contenant le gène, bientôt réduite à $250 \mathrm{~kb}$ entre D15S127 et D15S1108, sachant que les points de recombinaison observés étaient forcément intragéniques (figure 2). C'est chose faite et une partie de l'ADNc du gène $B L M$ vient d'être séquencée, dont l'extrémité 5'. La séquence nucléotidique identifiée a une forte analogie avec celles de gènes codant pour les hélicases de la famille RecQ; les acides aminés identiques sont concentrés dans la région qui contient les sept domaines hélicase conservés: $44 \%$ d'identité avec le produit du gène $R E C Q L$ humain isolé à partir de cellules HeLa qui possède une activité hélicase 3'-5' dépendante de l'ATP et de l'ADN, $43 \%$ avec SGS1 de Saccharomyces cerevisiae (qui interagit avec les topo-isomérases Top $2 p$ et Top3p et semble indispensable à la migration normale des chromosomes), et $42 \%$ avec recQ d'Escherichia coli (intervenant dans la voie $\mathrm{RecF}$ ) [6]. Ces trois gènes font partie de la sous-famille des hélicases à boîte DExH $(\mathrm{D}=\mathrm{Asp}, \mathrm{E}=\mathrm{Glu}, \mathrm{H}=\mathrm{His}$ et $\mathrm{x}=\mathrm{n}$ 'importe quel acide aminé). On sait que les hélicases ont la propriété d'ouvrir les deux brins d'ADN afin d'en assurer le déroulement, la lecture, ou les réparations $\left(\mathrm{m} / \mathrm{s} n^{\circ} 9 \mathrm{vol} .6\right.$, p. 924) [7]. Dans le xeroderma pigmentosum le syndrome de Cockayne et la trichothiodystrophie, les mutations des gènes ERCC (pour excision repair cross complementarity) empêchent la réparation de l'ADN $\left(m / s n^{\circ} 4\right.$, vol. 9 , p. 485, [2]). Dans le syndrome de Bloom, où il n'y a pas de trouble de réparation, les fonctions du produit du gène $B L M$ devraient donc concerner d'autres aspects du métabolisme de l'ADN.

La recherche de mutations chez 13 malades s'est révélée positive et les résultats très variés: substitution d'une base, insertion, délétion, codon non sens. On sait que la population d'origine juive ashkénase est particulièrement touchée par cette maladie. Les malades de cette population parmi les sujets de l'étude sont tous porteurs de la même mutation: une délétion de $6 \mathrm{pb}$, accompagnée d'une insertion de $7 \mathrm{pb}$, qui entraîne une rupture dans le cadre de lecture. Il s'agit vraisemblablement d'une mutation héritée d'un ancêtre commun puisqu'un déséquilibre de liaison y est associé [8]. Jusqu'à présent aucune différence n'a été observée dans le phénotype des malades, qu'ils soient homozygotes ou hétérozygotes composites et quelle que soit leur origine géographique.

Ces différentes mutations ont pour conséquence la suppression des activités enzymatiques de la protéine codée par le gène $B L M$. Outre des domaines hélicases, le transcrit contient des régions amino- et carboxy-terminales qui doivent lui conférer une activité spécifique: interaction avec une topo-isomérase, par exemple. On savait déjà que l'activité topo-isomérase II est diminuée dans les cellules des malades BS [9]. Quant à la survenue de cancers de tous types, elle n'est sans doute que la regrettable conséquence des recombinaisons mitotiques innombrables se produisant continûment sur l'ensemble du génome et favorisant toutes les recombinaisons illégitimes possibles.

Désormais, il nous tarde de connaître le rôle exact du facteur BLM et sa place dans la hiérarchie des facteurs 


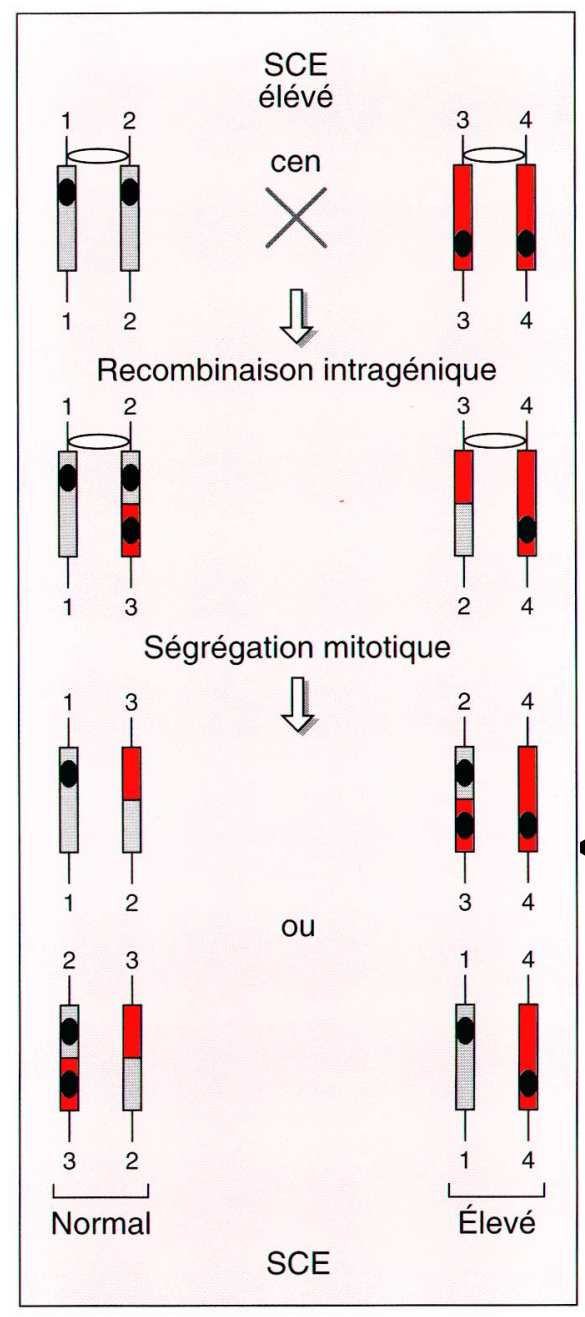

du métabolisme de l'ADN. Il est fondamental puisque ses mutations sont responsables de la maladie génétique qui est véritablement le prototype des maladies mutationnelles.

S.G.

1. Sarasin A. La réparation de l'ADN au centre de la biologie de la cellule. médecine/sciences 1994; 10: $951-2$.

2. Schaeffer L, Egly J. BTF2/TFIIH, un facteur de transcription et réparation impliqué dans des maladies de la réparation de l'ADN. médecine/sciences $1994 ; 10: 973-8$.

3. German J, Passarge E. Bloom's syndrome. XII. Report from the Registry for 1987. Clin Genet $1989 ; 35: 57-69$.

4. Ellis NA, Groden J, Ye TZ, Straughe J, Lennon DJ, Ciocci S, Proytcheva M, German J. The Bloom's syndrome gene product is homologous to RecQ helicases. Proc Acad Natl Sci USA 1994;

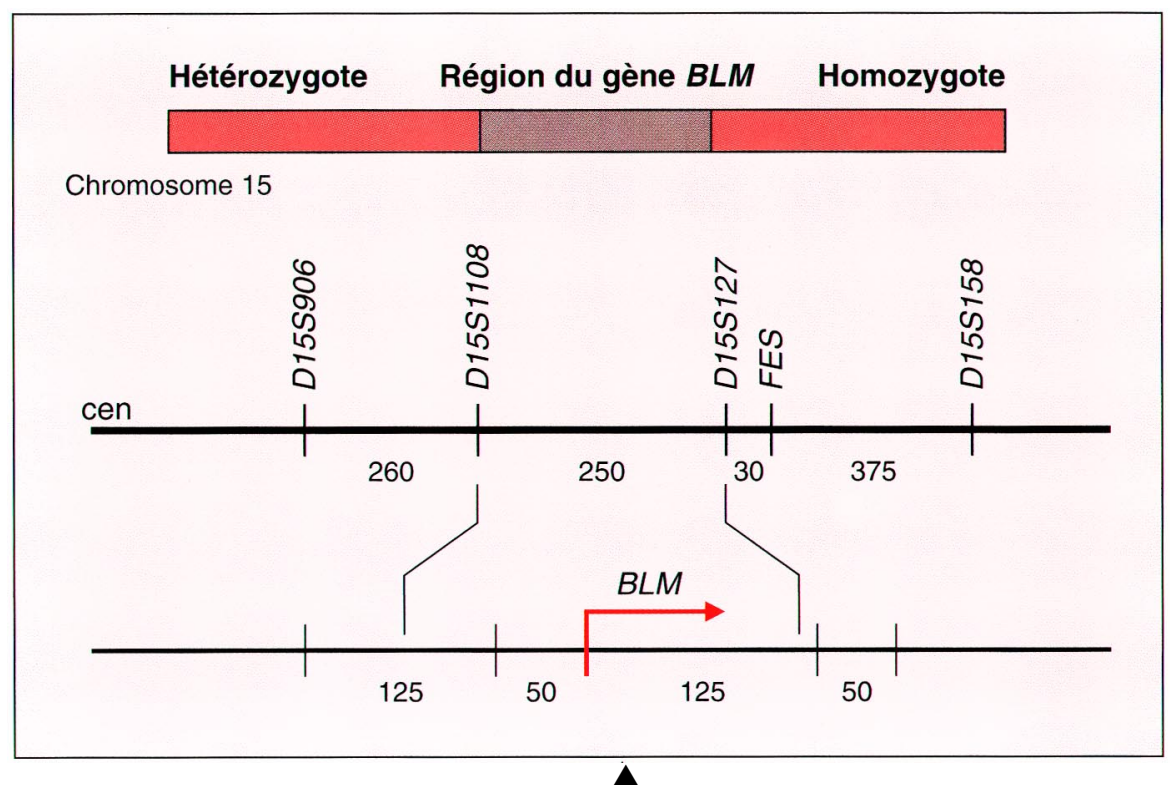

Figure 1. Modèle de recombinaison somatique intragénique. Les rectangles représentent le locus BLM, les cercles symbolisent les mutations. Les mutations sont situées à des sites différents sur les deux chromosomes. Au cours de la métaphase le locus BML peut être le lieu de recombinaison intragénique (croix). La perte de la mutation sur l'un des homologues normalise la fréquence des échanges de chromatides-sœurs (SCE). Cen: centromère. (D'après [10].)

Figure 2. Carte génétique de la région critique BLM établie grâce à la cartographie des points de recombinaison. $L^{\prime} A D N$ des cellules ayant une fréquence d'échange de chromatides-sœurs basse a été examiné: les marqueurs distaux par rapport à D15S127 étaient homozygotes, les marqueurs proximaux par rapport à D15S108 restaient hétérozygotes. La région comprise entre ces marqueurs, couvrant $250 \mathrm{~kb}$, contenait obligatoirement le gène BLM. Cen: centromère; les distances sont indiquées en kilobases. (D'après [10].)
5. Ellis NA, Lennon DJ, Proytcheva M, Alhadeff B, Henderson EE, German J. Somatic intragenic recombinaison within the mutated locus BLM can correct the high SCE phenotype of Bloom syndromes. Am J Hum Genet 1995; 57 : 1019-27.

6. Watt PM, Louis EJ, Borts RH, Hickson ID. Sgs1 : an eukaryotic homolog of E. Coli RecQ that interacts with topoisomerase II in vivo and is required for faithful chromosome segregation. Cell 1995; 81 : 253-60.

7. Toussaint C. Les hélicases. médecine/sciences $1995 ; 11: 1389-98$.

8. Ellis NA, Roe AM, Kozloski J, Proytcheva M, Falk C, German J. Linkage disequilibrium between the FES, D15S127, and BLM loci in Ashkenazi Jews with Bloom syndrome. Am J Hum Genet 1994; 55 : 453-60.

9. Heartlein MW, Tsuji H, Latt SA. 5-bromodeoxyuridine dependant increase in sister chromatid exchange formation in Bloom syndrome is associated with reduction in topoisomerase II activity. Exp Cell Res 1987; 169: 245-54.

10. Passarge E. A DNA helicase in full Bloom. $\mathrm{Na}$ ture Genet 1995 ; 11 : 356-7. 